\title{
A PSPICE Circuit Modeling of Strained AIGaInN Laser Diode Based on the Multilevel Rate Equations
}

\author{
Dong Wook Lim, Hyung Uk Cho, Hyuk Kee Sung, and Jong Chang Yi* \\ School of Electronic \& Electrical Engineering, Hongik University, Seoul 121-791, Korea \\ Young Min Jhon \\ Korea Institute of Science and Technology (KIST), Seongbuk, Seoul 136-791, Korea
}

(Received December 18, 2008 : revised August 28, 2009 : accepted September 10, 2009)

\begin{abstract}
PSPICE circuit parameters of the blue laser diodes grown on wurtzite AlGaInN multiple quantum well structures were extracted directly from the three level rate equations. The relevant optical gain parameters were separately calculated from the self-consistent multiband Hamiltonian. The resulting equivalent circuit model for a blue laser diode was schematically presented, and its modulation characteristics, including the pulse response and the frequency response, have been demonstrated by using a conventional PSPICE.
\end{abstract}

Keywords: AlGaInN blue LDs, Three-level rate equation, Equivalent circuit, PSPICE

OCIS codes: (230.0230) Optical devices; (140.2020) Diode lasers; (250.0250) Optoelectronics; (270.5580) Quantum electrodynamics

\section{INTRODUCTION}

The quaternary alloy AlGaInN has wide band gap energy, varying with the composition and covering almost all the visible spectrum, extending to the ultraviolet region. These nitride semiconductor technologies have led to the development of electrical devices such as optical data storage, mobile communication and medical surgery for applications in high power and high frequency regimes. The blue laser diode (LD) is an important device for such applications, especially for the optical pickup for the Blu-ray disc. An equivalent circuit model of blue LD, once developed, could be directly used in PSPICE for design and analysis of its driver circuitry. Currently available models presented electrical small signal models via RLC circuits including the spontaneous emission and gain saturation $[1,2]$. Others presented revised equivalent circuit models to accommodate the two-level and three-level rate equations: one equation explained the continuity of carrier charge in the quantum wells, anther described carrier charge in the separate-confinement heterostructure $(\mathrm{SCH})$, and the third recognized the gateway states introduced to

*Corresponding author: wave@hongik.ac.kr connect the carriers in $\mathrm{SCH}$ with the carriers in quantum wells [3,4]. In this paper, the PSPICE circuit parameters for blue LDs based on nitride compound semiconductors have been extracted from the threelevel rate equations. The relevant optical gain parameters were calculated separately from the self-consistent multiband Hamiltonian equations for the strained multiple quantum well (MQW) LD grown on wurtzite crystals [5]. AlGaInN Blue Laser Diodes are then described by the equivalent-circuit model for the design and analysis using PSPICE. This model provides a stable and accurate way of describing the LD modulation characteristics in both the large- and the small- signal analysis.

\section{THE THREE-LEVEL RATE EQUATION AND MODELING EQUIVALENT CIRCUIT}

The band structure of the blue LD to be modeled is as shown in FIG. 1. This AlGaInN SCH MQW LD structure is divided into three states for stable and accurate analysis. They are referred to as Separate confinement hetero-structure ( $\mathrm{SCH})$ state, Gateway $(\mathrm{G})$ state, and Quantum well $(\mathrm{QW})$ state. 


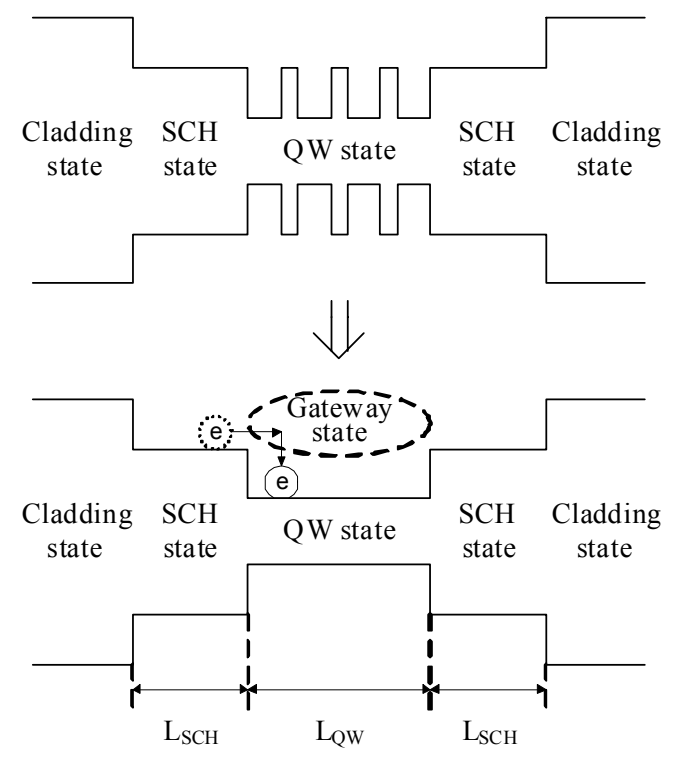

FIG. 1. The band structure of the AlGaInN Blue MQW LD.

The injected electrons and holes were captured in a quantum well after passing from the cladding state to the $\mathrm{SCH}$ state and the Gateway state. Most of the carriers captured in the QW were emitted via light and a few carriers are spilled out of the Quantum well by thermionic emission. Each state can be represented by the charges and the corresponding relaxation time constant. Therefore, the three-level rate equation of blue LD can be expressed as following: [4].

$$
\begin{aligned}
& \frac{d N_{S}}{d t}=\frac{I_{i}}{q}-\frac{N_{S}}{\tau_{n S}}-\frac{N_{S}}{\tau_{D}}+\frac{N_{G}}{\tau_{G}} . \\
& \frac{d N_{G}}{d t}=\frac{N_{S}}{\tau_{D}}-\frac{N_{G}}{\tau_{G}}-\frac{N_{G}}{\tau_{n G}}-\frac{N_{G}}{\tau_{C}}+\frac{N_{W}}{\tau_{E S C}} . \\
& \frac{d N_{W}}{d t}=\frac{N_{G}}{\tau_{C}}-\frac{N_{W}}{\tau_{E S C}}-\frac{N_{W}}{\tau_{n r W}}-\frac{N_{W}}{\tau_{r W}}-\frac{\Gamma v_{g} G\left(n_{W}\right) S}{1+\varepsilon_{p} S} . \\
& \frac{d S}{d t}=\frac{\Gamma v_{g} G\left(n_{W}\right) S}{1+\varepsilon_{p} S}-\frac{S}{\tau_{P}}+\beta \frac{N_{W}}{\tau_{r W}} .
\end{aligned}
$$

The $\tau_{r W}$ is the radiative recombination time constant in QW. $\tau_{n}$ 's are the nonradiative time constants for spontaneous recombination processes in each of Gateway, QW, and $\mathrm{SCH}$ states. $\tau_{D}$ is diffusion time constant in the $\mathrm{SCH}[4,6] . \tau_{C}$ is the ambipolar capture time constant for carriers transferring from the gateway states to the QW bound states [7]. $\tau_{G}$ is the time constant associated with the lifetime of carriers in the gateway states. $\tau_{E S C}$ is the thermionic emission life time [4]. $\tau_{P}$ is the photon lifetime which is given by $\tau_{p}=\left[v_{g} \times\left(a_{m}\right.\right.$ $\left.+a_{i n t}\right]^{-1}$ with $a_{m}=1 / L \times \ln (1 / R)$ and $a_{\text {int }}=12.1 \mathrm{~cm}^{-1}$ [8]. $I_{i}$ is the injection current, and $N_{S}, N_{G}, N_{W}$ are the carrier densities of $\mathrm{SCH}, \mathrm{G}$, and $\mathrm{QW}$, respectively. $\Gamma$ is the optical confinement factor [9]. $\beta$ is the spontaneous emission factor, $\mathrm{G}\left(n_{W}\right)$ is the carrier-densitydependent material gain. $v_{g}$ is the group velocity. Equation (1) denotes that the charge variation in $\mathrm{SCH}$ state is the injection current plus transferred carriers from Gateway state to SCH state, minus transferred carriers from $\mathrm{SCH}$ state to Gateway state and transferred carriers from $\mathrm{SCH}$ state to ground state because of electron-hole recombination. Similarly, equation (2) denotes the charge variation in the Gateway state and equation (3) presents the charge variation in the $\mathrm{QW}$ state. This rate equation can be simplified as by Benjamin $[10]$.

$$
\begin{aligned}
& I_{i}=\frac{d Q_{S}}{d t}+\frac{Q_{S}}{\tau_{n S}}+\frac{Q_{S}}{\tau_{D}}-\frac{Q_{G}}{\tau_{G}} . \\
& \frac{Q_{S}}{\tau_{D}}-\frac{Q_{G}}{\tau_{G}}=\frac{d Q_{G}}{d t}+\frac{Q_{G}}{\tau_{n G}}+\frac{Q_{G}}{\tau_{C}}-\frac{Q_{W}}{\tau_{E S C}} . \\
& \frac{Q_{G}}{\tau_{C}}-\frac{Q_{W}}{\tau_{E S C}}=\frac{d Q_{W}}{d t}+\frac{Q_{W}}{\tau_{r W}}+\frac{Q_{W}}{\tau_{n W}}+\frac{\Gamma q v_{g} G\left(n_{Q}\right) S}{\sqrt{1+\varepsilon_{p} S}} . \\
& q \frac{d S}{d t}=\frac{q \Gamma v_{g} G\left(n_{Q}\right) S}{\sqrt{1+\varepsilon_{p} S}}-q \frac{S}{\tau_{P}}+\beta \frac{Q_{W}}{\tau_{r W}} .
\end{aligned}
$$

The density of carriers and photons in the three-level rate equations described above are translated into voltages and currents as in Fig. 2. Here "G" represents the dependent current. $G_{1}, G_{2}, G_{3}, G_{4}$ are each $\tau_{n S}$. $Q_{S} / \tau_{n S} \cdot \tau_{D}, \tau_{n G} \cdot{ }_{Q G} / \tau_{n G} \cdot \tau_{G}, \tau_{n G} \cdot{ }_{Q G} / \tau_{n G} \cdot \tau_{C}, \tau_{n W} \cdot$ $Q_{W} / \tau_{n W} \cdot \tau_{E S C}$. The spontaneous photon emission and stimulated photon emission are determined by the dependent current source Gspon and Gstim, respectively

$$
\begin{aligned}
& G_{\text {stim }}=\frac{q \Gamma v_{g} G\left(n_{Q}\right) V_{\text {out }}}{\bar{p} \sqrt{1+\varepsilon_{p} V_{\text {out }} / \bar{p}}}, \quad R_{P}=\frac{\tau_{P} \bar{p}}{q}, \quad C_{P}=\frac{q}{\bar{p}} . \\
& V_{\text {out }}(V) \equiv P_{\text {out }}(W) \equiv \bar{p} S, \quad \bar{p}=\frac{1}{2} h v v_{g} \frac{1}{L_{L}} \ln \frac{1}{R} .
\end{aligned}
$$

Where $\bar{p}$ is a constant for converting photon population. $\varepsilon_{p}$ is the gain suppression factor for nonlinear

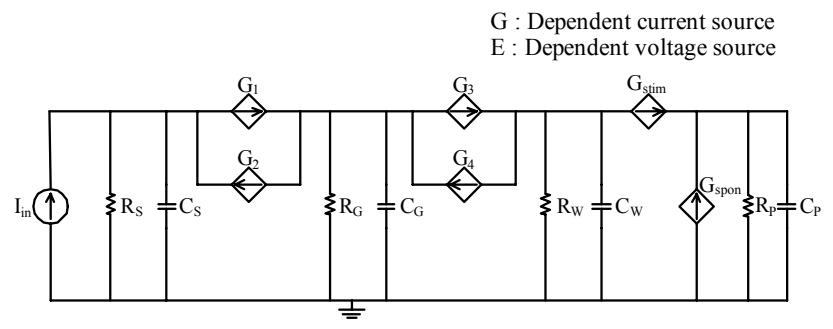

FIG. 2. PSPICE equivalent circuit model derived from the three-level rate equations. 
optical gain properties. This nonlinear gain factor is partially caused by the depletion of electrons at certain energy levels due to strong stimulated recombination. $h$ is Planck's constant, $L_{L}$ is the cavity length, and $\mathrm{R}$ is the end-face reflectivity [10].

\section{BLUE LASER DIODE PARAMETER EXTRACTIONS}

The $405 \mathrm{~nm}$ laser diode under investigation has typical AlGaInN MQW active structures as shown in FIG. 3. The quantum well regions are $3 \mathrm{~nm} \operatorname{In}_{0.12} \mathrm{Ga}_{0.88} \mathrm{~N}$ and the barrier regions are $\mathrm{In}_{0.02} \mathrm{Ga}_{0.98} \mathrm{~N}$. The $\mathrm{SCH}$ regions are $\mathrm{Al}_{0.2} \mathrm{Ga}_{0.8} \mathrm{~N}$ and the cladding regions are $\mathrm{N}$ and P-doped $\mathrm{Al}_{0.13} \mathrm{Ga}_{0.87} \mathrm{~N}$ sandwiched by $\mathrm{GaN}$ ohmic contact layers [11].

FIG. 4. shows the energy band diagram of multiple quantum well (MQW) and electron blocking layer (EBL). For the enhanced optical performance, P-doped $\mathrm{Al}_{0.3} \mathrm{Ga}_{0.7} \mathrm{~N}$ EBL is indispensable for the suppression of electron overflow from the active layer to the p-type cladding layer. The gain and spontaneous emission spectra of the AlGaInN QW LD are obtained by a

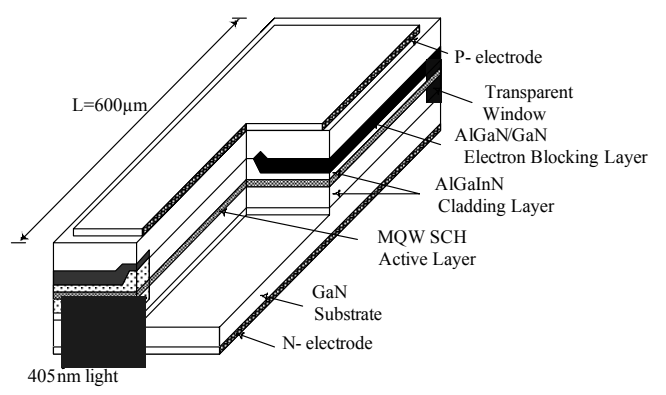
LD.

FIG. 3. Schematic perspective of the AlGaInN MQW

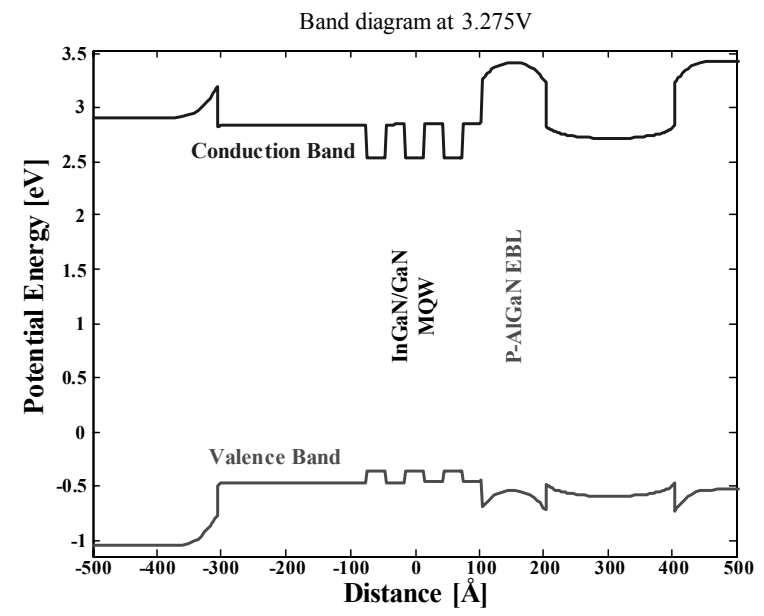

FIG. 4. Band profiles of the MQW and EBL regions obtained by the self-consistent analysis. numerical analysis of self-consistent Poisson equations and the Hamiltonian equations for multiband strained wurtzite multiple quantum well structures [12].

FIG. 5 shows gain spectra for the various carrier injections from $1.8 \times 10^{19}\left[\mathrm{~cm}^{-3}\right]$ to $5.0 \times 10^{19}\left[\mathrm{~cm}^{-3}\right]$ in 20 steps. FIG. 6 shows the variation of maximum material gain $\left(G_{\max }\right)$ and the differential gain $\left(G_{N}\right)$ due to carrier density.

The gain was calculated using the $k$-selection rule, taking into account the intraband relaxation broadening. An excellent fit to the results can be obtained with the following expression $[4,5]$.

$$
G\left(n_{W}\right)=G_{0} \times\left(n_{W}-G_{1}\right)+G_{2} .
$$

where $G_{0}$ is the optical-gain coefficient and $G_{1}, G_{2}$ are the carrier density for transparency [13]. Other parameters were also extracted by following the procedures described in as shown in TABLE 1. [3-14].

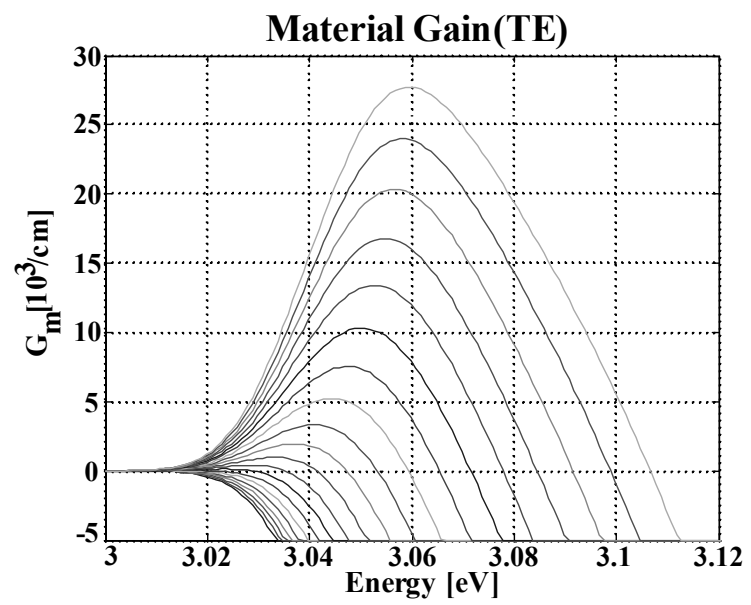

FIG. 5. Gain spectra for the various carrier injections from $1.8 \times 10^{19}$ to $5.0 \times 10^{19} \mathrm{~cm}^{-3}$ in 20 steps.
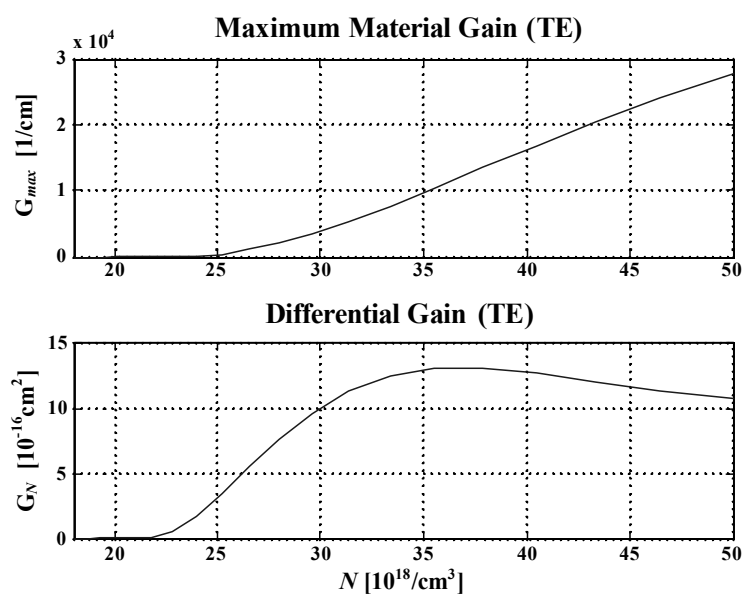

FIG. 6. Maximum material gain $\left(\mathrm{G}_{\max }\right)$ and Differential Gain $\left(\mathrm{G}_{\mathrm{N}}\right)$ of AlGalnN MQW LD. 


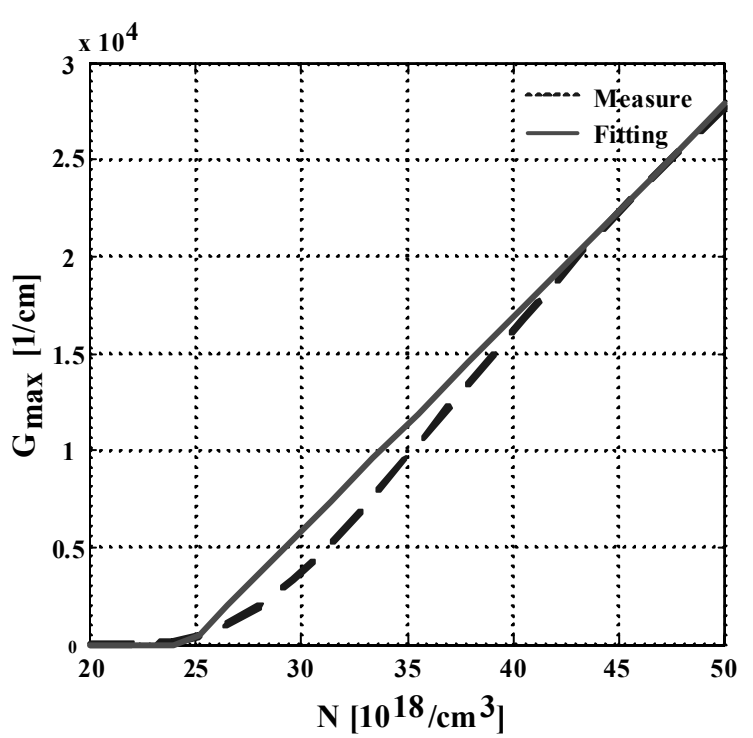

FIG. 7. The Maximum Material Gain Fitting of the AlGaInN Blue MQW LD.

TABLE 1. Model parameter values used in the simulation

\begin{tabular}{c|c|c|c}
\hline \hline Parameter & Unit & Value & Source \\
\hline$\tau_{p}$ & $\mathrm{ps}$ & 2.24 & {$[8]$} \\
\hline$\tau_{n S}$ & $\mathrm{~ns}$ & 1 & {$[4]$} \\
\hline$\tau_{n G}$ & $\mathrm{~ns}$ & 1 & {$[4]$} \\
\hline$\tau_{n W}$ & $\mathrm{~ns}$ & 1.1 & {$[14]$} \\
\hline$\tau_{r G}$ & $\mathrm{~ns}$ & 1 & {$[12]$} \\
\hline$\tau_{D}$ & $\mathrm{ps}$ & 113.12 & {$[4,6]$} \\
\hline$\tau_{G}$ & $\mathrm{ps}$ & 1 & {$[4]$} \\
\hline$\tau_{C}$ & $\mathrm{ps}$ & 0.2 & {$[7]$} \\
\hline$\tau_{E S C}$ & $\mathrm{ps}$ & 3.02 & {$[8]$} \\
\hline$v g$ & $\mathrm{~ms}$ & $1.15 \times 10^{8}$ & {$[3]$} \\
\hline$\Gamma$ & & 0.017 & see text \\
\hline$\beta$ & & $1 \times 10^{-4}$ & see text \\
\hline$\varepsilon_{p}$ & & $1 \times 10^{-7} 1 \times 10^{-9}$ & see text \\
\hline$G_{0}$ & $\mathrm{~cm}^{2}$ & $1.1 \times 10^{-15}$ & see text \\
\hline$G_{1}$ & $\mathrm{~cm}^{-3}$ & $2.5 \times 10^{19}$ & see text \\
\hline$G_{2}$ & $\mathrm{~cm}^{-1}$ & 371 & see text \\
\hline $\bar{p}$ & $\mathrm{~W}$ & $7.6 \times 10^{-8}$ & see text \\
\hline$R_{P}$ & $\mathrm{~W}$ & 1.064 &
\end{tabular}

\section{SIMULATION RESULTS AND DISCUSSION}

The simulation results show full characteristics of blue LD in small-signal and large-signal regimes. First, the pulse response was shown in FIG. 8, where it shows the existence of a few ns turn-on delay and a relaxation oscillation when increasing the pulse signal. The damping of the oscillations, determined by the gain

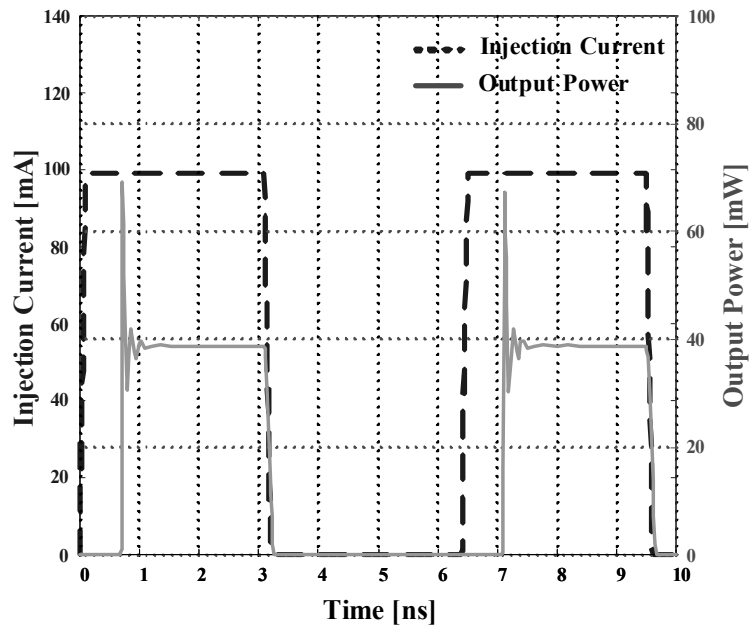

FIG. 8. PSPICE pulse response of the blue MQW LD.

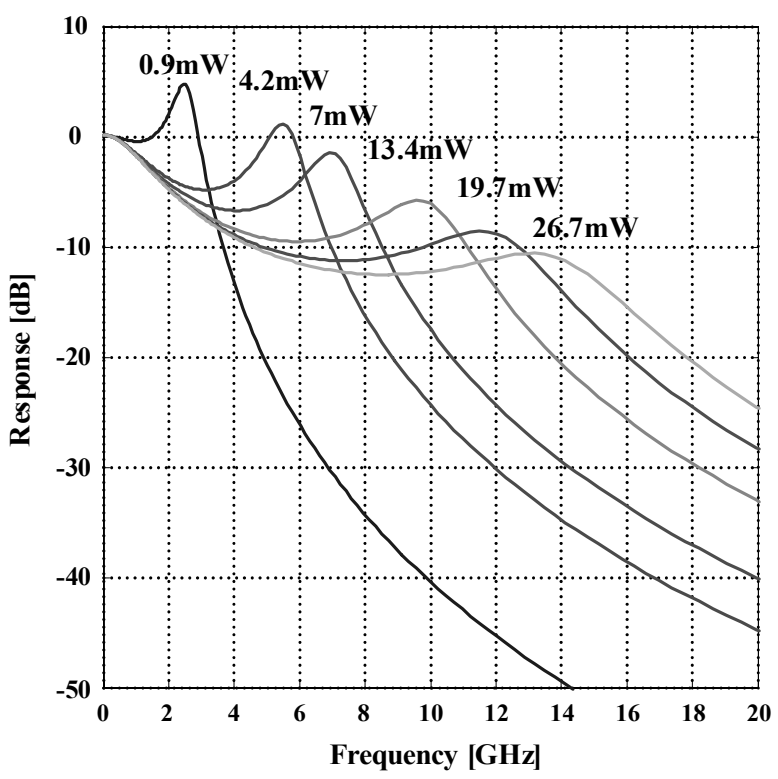

FIG. 9. Frequency response of the blue LD for various output power levels $\left(\varepsilon_{p}=1 \times 10^{-7} \mathrm{~m}^{3}\right)$.

suppression factor, can also be recognized.

The frequency response characteristics of the blue LD are also shown in FIG. 9 and FIG. 10 for two different values of the gain suppression factors. Both the figures show the increase of the $3 \mathrm{~dB}$ bandwidth according to the optical power increase. Furthermore, the maximum peak values of the responses are decreased by increasing the optical power. Such a characteristic behavior in the frequency response has been predicted elsewhere which takes into account the presence of gateway states $[4,6]$. FIG. 11 shows the effect of the gain suppression factor on the modulation characteristics, which shows a decrease in the modulation bandwidth due to the over damping $[4,15]$. The V-I characteristics curve for equivalent circuit 


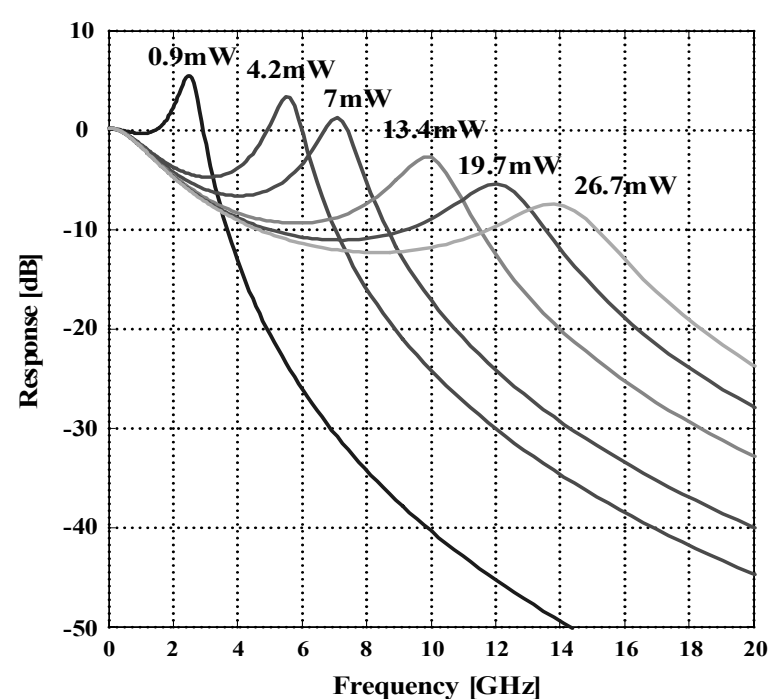

FIG. 10. Frequency response of the blue LD for various output power levels $\left(\varepsilon_{p}=1 \times 10^{-9} \mathrm{~m}^{3}\right)$.

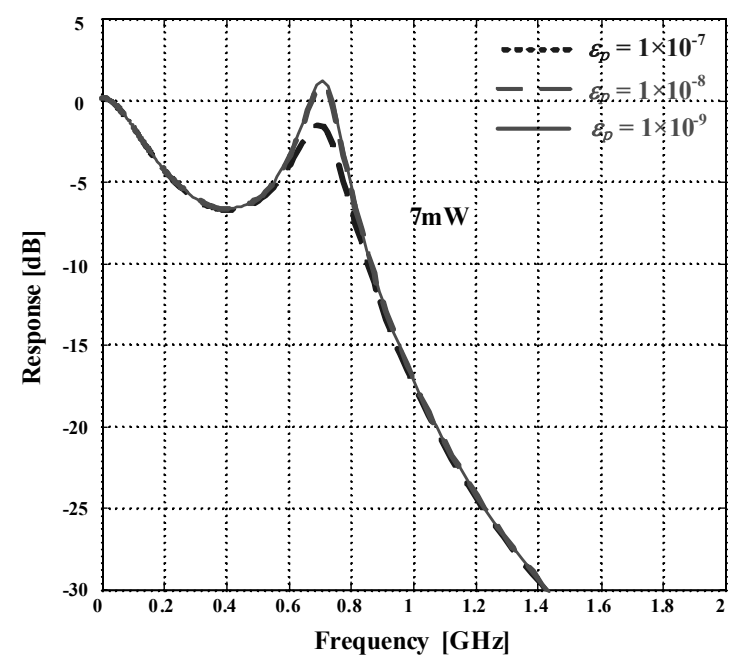

FIG. 11. Effect of the gain suppression factor on the frequency response of the blue $\mathrm{LD}(14.8 \mathrm{~mW})$.

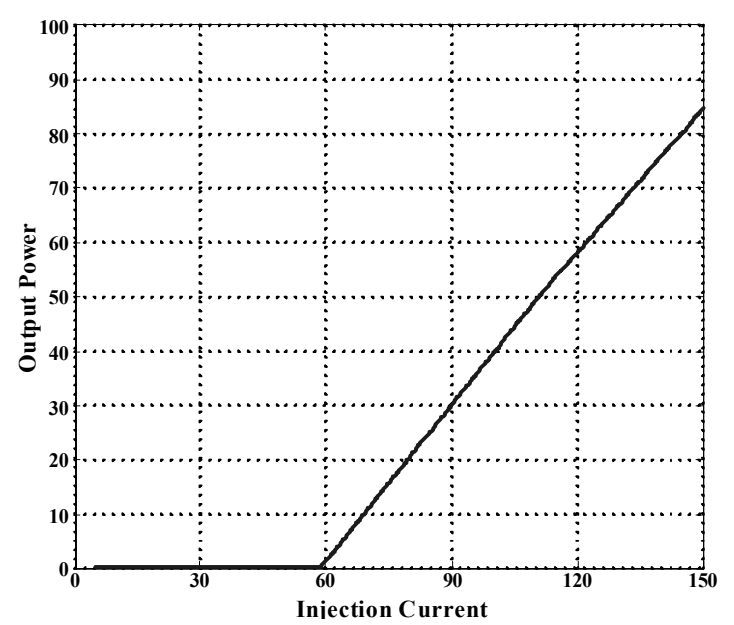

FIG. 12. V-I characteristics curve for equivalent circuit model of Blue LD. model of Blue LD are shown in FIG. 12. This figure shows that the threshold current of blue LD is $60 \mathrm{~mA}$.

\section{CONCLUSION}

In this work, the modulation characteristics of AlGaInN blue laser diodes were described by an equivalent-circuit model for the conventional PSPICE. This model is based on the three-level rate equations that include the role of gateway states at the quantum well in their characterization of charge dynamics. The device parameters were extracted from the optical gain properties of the MQW active region using the selfconsistent Poisson equation and the multiband Hamiltonian for the strained wurtzite crystal. The resulting model is schematically presented and the modulation characteristics were proved by using the convention SPICE, which shows all the key features expected in MQW LD operation such as modulation bandwidth dependence on injection current and turn-on delay, the relaxation oscillation, as well as the over damping effect due to the gain suppression. Such an equivalent circuit model is expected to be directly applicable for optical data storage, mobile communication and medical machinery.

\section{ACKNOWLEDGMENT}

This work was supported by the Korea Science and Engineering Foundation (KOSEF) grant funded by the Korea government (MOST) under R01-2007-000-20048-0

\section{REFERENCES}

1. M. Morishita, T. Ohmi, and J. Nishizawa, "Impedance characteristics of double heterostructure laser diodes," Solid State Electron., 22, 951-962 (1979).

2. J. Katz, S. Margalit, C. Harder, D. Wilt, and A. Yariv, "The intrinsic electrical equivalent circuit of a laser diode,” IEEE J. Quantum Electron. 17, 4-7 (1981).

3. L. V. T. Nguyen, A. J. Lowery, P. C. R. Gurney, and D. Novak, "A time-domain model for high-speed quantumwell lasers including carrier transport effects," IEEE J. Quantum Electron. 1, 494-504 (1995).

4. D. McDonald and R. F. O'Dowd, "Comparison of two-and three-level rate equations in the modeling of quantumwell lasers," IEEE J. Quantum Electron. 31, 1927-1934 (1995).

5. J. C. Yi and N. Dagli, "Finite-element analysis of valence band structure and optical properties of quantum-wire arrays on vicinal substrates," IEEE J. Quantum Electron. 31, 208-218 (1995).

6. R. Nagarajan, M. Ishikawa, T. Fukushima, R. S. Geels, and J. E. Bowers, "High speed quantum-well lasers and carrier transport effects," IEEE J. Quantum Electron. 
28, 1990-2008 (1992).

7. W. H. Fan, S. M. Olaizola, J.-P. R. Wells, D. J. Mowbray, M. S. Skolnick, A. M. Fox, T. Wang, and P. J. Parbrook, "Electron capture time in InGaN/GaN multiple quantum wells," in Proc. Conference on Lasers and Electro-optics (San Francisco, California, USA, May 2004), CD, paper CThV2.

8. J. Piprek, Semiconductor Optoelectronic Devices (Academic Press, University of California at Santa Barbara, USA, 2003), Chapter 9.

9. T. Meyer, H. Braun, U. T. Schwarz, S. Tautz, M. Schillgalies, S. Lutgen, and U. Strauss, "Spectral dynamics of $405 \mathrm{~nm}(\mathrm{Al}, \mathrm{In}) \mathrm{GaN}$ laser diodes grown on GaN and SiC substrate," Opt. Exp. 16, 6833-6845 (2008).

10. B. P. C. Tsou and D. L. Pulfrey, "A versatile SPICE model for quantum-well lasers," IEEE J. Quantum Electron. 33, 246-254 (1997).
11. S. Nagahama, T. Yamamoto, M. Sano, and T. Mukai, “Blue-violet nitride laser," Phys. Stat. Sol. (a) 194, 423-427 (2002).

12. H. U. Cho and J. C. Yi, "External feedback effects on the relative intensity noise characteristics of InAlGaN blue laser diodes," J. Opt. Soc. Korea 10, 86-90 (2006).

13. G. Jiamjun, G. Baoxin, and L. Chunguang, "Large signal model of quantum-well lasers for SPICE," Microwave and Optical Technology Letters 39, 295-298 (2003).

14. C. Netzel, R. Doloca, S. Lahmann, U. Rossow, and A. Hangleiter, "Radiative and nonradiative recombination times in optically excited GaInN/GaN quantum wells," Phys. Stat. Sol. (c) 0, 324-328 (2002).

15. M. Grupen, G. Kosinovsky, and K. Hess, "The effect of carrier capture on the modulation bandwidth of quantum well lasers," in Proc. Electron Devices Meeting (Washington, USA, Dec. 1993), pp. 609-612. 